\title{
BMJ Open Study protocol for the SARON trial: a multicentre, randomised controlled phase III trial comparing the addition of stereotactic ablative radiotherapy and radical radiotherapy with standard chemotherapy alone for oligometastatic non-small cell lung cancer
}

\author{
John Conibear, ${ }^{1}$ Brendan Chia, ${ }^{1}$ Yenting Ngai, ${ }^{2}$ Andrew Tom Bates, ${ }^{3}$ \\ Nicholas Counsell, ${ }^{2}$ Rushil Patel, ${ }^{4}$ David Eaton, ${ }^{4}$ Corinne Faivre-Finn, ${ }^{5}$ \\ John Fenwick, ${ }^{6}$ Martin Forster, ${ }^{7}$ Gerard G Hanna, ${ }^{8}$ Susan Harden, ${ }^{9}$ Philip Mayles, ${ }^{10}$ \\ Syed Moinuddin, ${ }^{7}$ David Landau ${ }^{11,12}$
}

To cite: Conibear J, Chia B, Ngai Y, et al. Study protocol for the SARON trial: a multicentre, randomised controlled phase III trial comparing the addition of stereotactic ablative radiotherapy and radical radiotherapy with standard chemotherapy alone for oligometastatic non-small cell lung cancer. BMJ Open 2018;8:e020690. doi:10.1136/ bmjopen-2017-020690

- Prepublication history and additional material for this paper are available online. To view these files, please visit the journal online (http://dx.doi. org/10.1136/bmjopen-2017020690).

A poster abstract of this study was published at the IASLC 17th World Conference on Lung Cancer.

Received 1 December 2017 Revised 2 February 2018 Accepted 9 February 2018

Check for updates

(c) Author(s) (or their employer(s)) 2018. Re-use permitted under CC BY. Published by BMJ.

For numbered affiliations see end of article.

Correspondence to

Dr David Landau;

david.landau@kcl.ac.uk

\section{ABSTRACT}

Introduction Following growing evidence to support the safety, local control (LC) and potential improvement in overall survival (OS) in patients with oligometastatic nonsmall cell lung cancer (NSCLC) that have been treated with local ablative therapy such as stereotactic ablative radiotherapy (SABR) and stereotactic radiosurgery (SRS), we initiate the SARON trial to investigate the impact and feasibility of adding SABR/SRS and radical radiotherapy (RRT) following standard chemotherapy on OS.

Methods and analysis SARON is a large, randomised controlled, multicentre, phase III trial for patients with oligometastatic EGFR, ALK and ROS1 mutation negative NSCLC (1-3 sites of synchronous metastatic disease, one of which must be extracranial). 340 patients will be recruited over 3 years from approximately 30 UK sites and randomised to receive either standard platinumdoublet chemotherapy only (control arm) or standard chemotherapy followed by RRT/SABR to their primary tumour and then SABR/SRS to all other metastatic sites (investigational arm). The primary endpoint is OS; the study is powered to detect an improvement in median survival from 9.9 months in the control arm to 14.3 months in the investigational arm with $85 \%$ power and two-sided $5 \%$ significance level. The secondary endpoints are LC, progression-free survival, new distant metastasisfree survival, toxicity and quality of life. An early feasibility review will take place after 50 randomised patients. Patients requiring both conventional thoracic $\mathrm{RT}$ to the primary and SABR to a thoracic metastasis will be included in a thoracic SABR safety substudy to assess toxicity and planning issues in this subgroup of patients more thoroughly.

Ethics and dissemination All participants are given a SARON patient information sheet and required to give written informed consent. Results will be submitted for

\section{Strengths and limitations of this study}

- SARON is a large, randomised controlled, multicentre, UK phase III trial.

- 340 patients will be recruited over 3 years from approximately 30 UK sites.

- The trials primary endpoint is overall survival and is powered to detect an improvement in median survival from 9.9 months in the control arm to 14.3 months in the experimental arm.

- Patients requiring both conventional thoracic radiotherapy to the primary and stereotactic ablative radiotherapy (SABR) to a thoracic metastasis will be included in a thoracic SABR safety substudy to assess toxicity and planning issues in this unique and challenging subgroup of patients more thoroughly.

- The trial will undergo an early feasibility review after 50 randomised patients to ensure full recruitment is likely to be achievable.

presentation at local and international conferences and expected to be published in a peer-reviewed journal.

Trial registration number NCT02417662.

Sponsor reference UCL/13/0594.

\section{BACKGROUND}

Oligometastatic NSCLC

Unselected European patients with oligometastatic non-small cell lung cancer (NSCLC) treated with cytotoxic chemotherapy have a median survival of 8.5-10.5 months. ${ }^{12}$ This figure includes patients with any number of metastases. There are increasing data though 
showing that patients with fewer sites of metastases seem to have a better overall survival (OS).

The MD Anderson group reported a retrospective review of outcomes in 1284 patients with metastatic NSCLC. ${ }^{3}$ It reported that patients with a single organ involved site had better OS than those with two or more. In 137 patients with lung metastases only, the risk of death was correlated with the number of metastases. Another study, the FLEX study, enrolled 1125 patients with metastatic NSCLC and reported similar OS benefit in patients with metastases in one site versus two sites versus three or more sites (12.4 months vs 9.8 months vs 6.4 months, $\mathrm{p}<0.001) .{ }^{4}$ Similarly, the Southwest Oncology Group published an analysis of prognostic factors in 2531 patients enrolled in 14 phase II-III trials. ${ }^{5}$ The results confirm the prognostic significance of single versus multiple metastases on multivariate analysis. These studies support the hypothesis that a lower tumour burden is associated with improved outcome.

The concept of oligometastatic disease was established in an editorial by Hellman and Weichselbaum. ${ }^{6}$ In it, they described that patients with a lower tumour burden might be candidates for a more radical approach to management. The key issues needed were the definitive demonstration that alternative treatment strategies would improve patient outcomes and the ability to prospectively identify patients with the least propensity to develop further systemic metastases.

\section{Existing knowledge}

Numerous retrospective studies ${ }^{7}$ have been published establishing the safety and efficacy of stereotactic ablative radiotherapy $(\mathrm{SABR}) /$ stereotactic radiosurgery (SRS) in achieving durable local control (LC) in many different single and multiorgan sites (lung, ${ }^{8}$ spine,${ }^{9}$ liver,${ }^{10-12}$ lymph nodes, ${ }^{13-15}$ adrenal glands, ${ }^{1617}$ multiple metastases in individual organs (liver, ${ }^{10-12}$ lung $^{1819}$ or multiple organs ${ }^{1920}$ ).

In metastatic NSCLC, approximately $44 \%$ of patients will have brain metastases at first diagnosis. ${ }^{21}$ There is now evidence to suggest though that aggressive Central Nervous System directed treatment improves progression-free survival (PFS) and/or OS in some patients. ${ }^{22}$

Table 1 summarises the results of some of these SABR/ SRS studies and highlights the 1-year and 2-year control rates and low incidence of grade $3+$ toxicities seen so far in the literature.

A few prospective studies have also been reported. De Ruysscher et al reported a single-arm phase II trial investigating whether it would be possible to obtain a significant 2-year and 3-year survival in patients when all macroscopic sites in oligometastatic NSCLC are treated with radical RT. ${ }^{21}$ The median OS was 13.5 months and PFS was 12.1 months. In comparison, PFS in systemic therapy-only trials is reported to be around 4-6 months. ${ }^{1}$ Interestingly, the 2-year and 3-year PFS were maintained at 13.6\%.

Another report is by Holy et $a l^{16}$ treating patients with NSCLC and adrenal metastases. Median PFS was 4.2 months for the entire patient group, but in those with isolated adrenal metastases, the PFS was 12 months. At
21 months median follow-up, 10 of 13 patients (77\%) with an isolated adrenal metastasis maintained LC with a median OS of 23 months.

Recently, Gomez and colleagues published the first, randomised trial supporting the first point made by Hellman and Weichselbaum. ${ }^{83}$ The trial was a phase II, multicentre study which randomised patients with NSCLC with oligometastatic disease (defined as $\leq 3$ metastases) who did not progress after first line systemic treatment to either local consolidative therapy to all metastases, with or without systemic therapy or to standard systemic therapy alone. Local consolidative treatments included surgery, RT, chemo-RT or a combination thereof. The study was closed early after 49 patients were enrolled, as interim analysis found the median PFS in the local consolidative therapy arm to be 14.4 months compared with 3.9 months in the standard systemic therapy arm. ${ }^{23}$ Median OS was not reached. Recently, another phase II trial was reported by Texas Southwestern Medical Centre. ${ }^{24}$ They enrolled 29 patients with similar enrolment criteria, but their study design allowed for $\leq 6$ sites of metastatic disease. The study was stopped early as interim analysis showed a significant PFS advantage in the SABR arm (9.7 vs 3.5 months). SABR resulted in no in-field failure vs seven in the maintenance only arm. No additional toxic effect was noted in the SABR arm and median OS was also not reached.

There are several ongoing trials, either recruiting or in planning, such as ROLE (NCT01796288) and CORE (NCT02759783) that could support the role of radical radiotherapy (RRT)/SABR in oligometastatic disease further.

\section{Need for a trial}

Currently there is no internationally agreed management strategy of oligometastatic NSCLC. Management recommendations are thus widely variable and depend on local and patient-specific factors.

A large randomised phase III trial specific to NSCLC is therefore needed to ascertain if the addition of RRT with SABR/SRS or conventional radiotherapy to chemotherapy is safe and effective in improving the outcomes of patients with oligometastasis.

\section{Choice of comparator}

Without the ability to predict lack of systemic progression, the standard first-line management for patients with oligometastatic NSCLC remains to be chemotherapy.

In the SARON trial, the control arm will therefore be four cycles of platinum-based doublet chemotherapy followed by maintenance systemic therapy as per local policy. The type and dose regimen of chemotherapy given will be based on institution protocols.

Patients with sensitising EGFR, ALK-fusion and ROS1 mutations have a different natural history and outcome to those without. ${ }^{33}$ To maintain as homogeneous a population as possible, these patients are excluded from SARON. 
Table 1 Summary of referenced studies of SABR in the treatment of oligometastatic disease

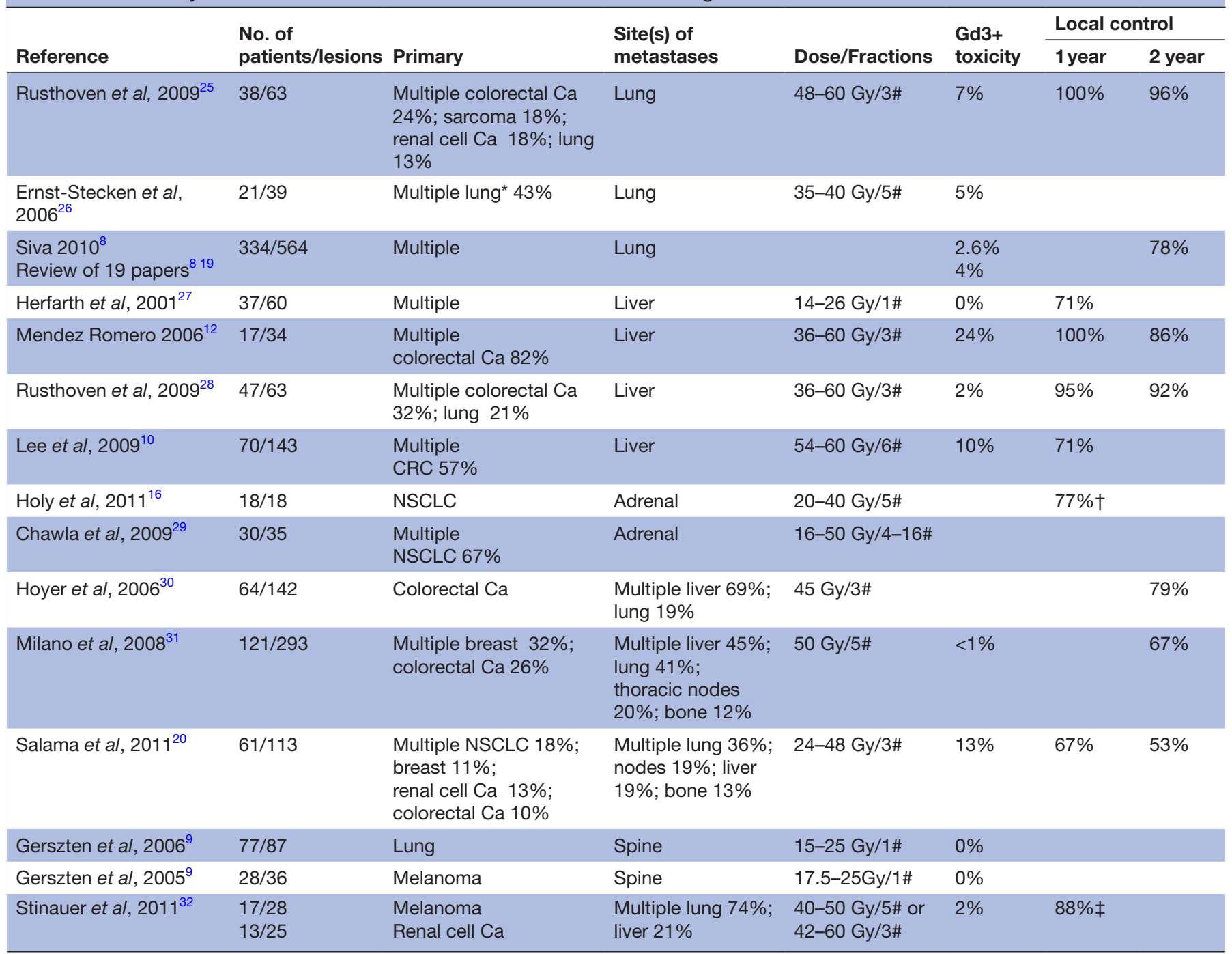

*These were primary lung cancers.

†At a median follow-up of 21 months.

$\ddagger$ At 18 months.

NSCLC, non-small cell lung cancer; SABR, stereotactic ablative radiotherapy.

\section{STUDY OBJECTIVES AND HYPOTHESIS \\ Study hypothesis}

We propose that radical treatment with SRS/SABR or conventional radiotherapy to the primary lung and metastatic lesions is safe, feasible and effective in improving the survival outcomes of oligometastatic NSCLC that have responded to initial treatment with systemic chemotherapy.

\section{Primary objective}

To investigate the impact the addition RRT using SABR/ SRS or conventional RT has on OS in oligometastatic NSCLC that are treated with first-line standard systemic therapy.

\section{Secondary objectives}

To demonstrate the impact of a RRT strategy on:

- Progression-free survival
- Includes loss of LC of target lesions and development of new distant metastases which will be recorded separately.

- Also includes non-measurable radiological progression.

- Toxicity

- RT-related toxicity.

- Early and late toxicity.

- LC of primary and all metastases (refers to all lesions present at time of randomisation. A record will be kept of primary versus metastatic progression)

- Quality of life

\section{Feasibility substudy}

To demonstrate that the recruitment and withdrawal rates are consistent with achieving the aims of the trial and that there are no unforeseen logistical challenges 


\section{SUB-STUDIES WITHIN OVERALL STUDY}

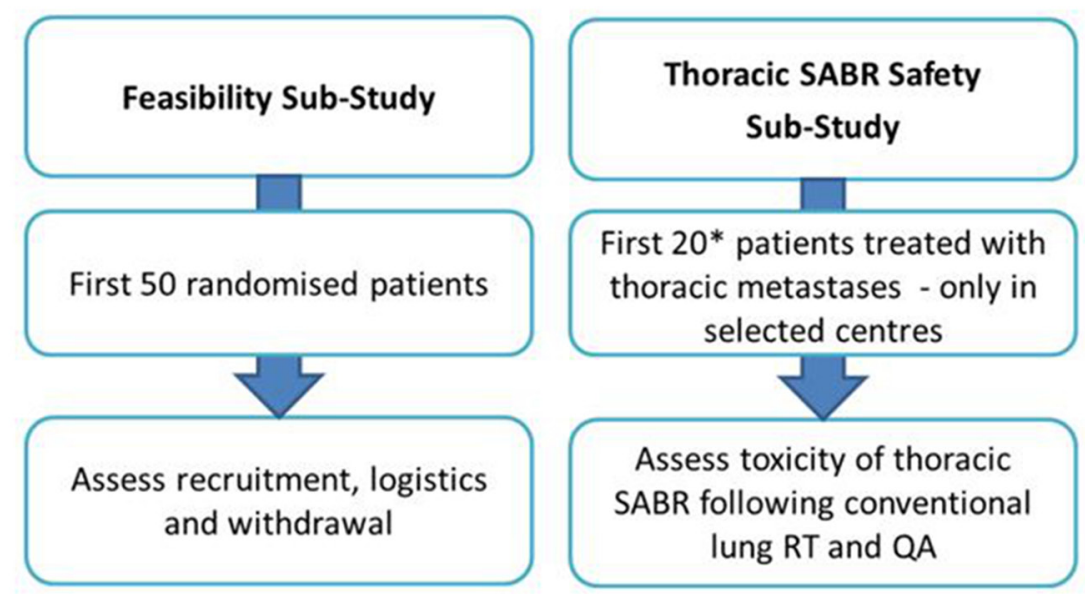

* More patients may be recruited if required to confirm safety

Figure 1 SARON substudy schema. QA, quality assurance; RT, radiotherapy; SABR, stereotactic ablative radiotherapy.

which would prevent achieving full recruitment within the timescales set out for the study.

\section{Thoracic SABR safety substudy}

This will be done to document the toxicity and feasibility of delivering SABR to thoracic metastases with or immediately following radical thoracic RT. Additionally, we will be able to assess processes for RT planning and dosimetry and have quality assurance $(\mathrm{QA})$ for this group of patients.

\section{TRIAL DESIGN AND METHODS}

SARON is a randomised, multicentre, non-blinded, parallel-phase III trial (including trial feasibility and thoracic metastatic SABR safety components) for patients with oligometastatic (1-3 metastases) NSCLC. As already stated, the SARON trial will also include two substudies on feasibility and thoracic radiation safety (figure 1).

\section{Main trial}

Patients will be randomised in a 1:1 ratio to receive either standard systemic therapy only (Control Arm) or standard systemic therapy plus radical RT or SABR to their primary tumours (and N1-3 mediastinal nodes where present) and SABR/SRS to all the metastatic deposits (Investigational Arm) (figure 2).

Allocation to either arm will be decided by a computer-generated randomisation schedule. Patients will be stratified by investigational site (hospital), histology (adenocarcinoma vs non-adenocarcinoma), nodal stage (N0/1 vs N2/3), number of oligometastatic sites (1 vs 2 or 3 ), brain metastases (present vs absent).

Due to the different treatment modalities in the study, it is not possible to blind the patient or the physician to the treatment arm.

\section{Setting}

The trial will be held in the UK with a target of 30 hospitals/cancer centres that are able to give chemotherapy and conventional RRT and to provide SABR/SRS (whether in their own centre or via referral to another centre). All sites giving RT must have QA accreditation, as guided by the Radiotherapy Treatment Quality Assurance (RTTQA) group of the National Cancer Research Institute (NCRI).

Cancer Research UK \& UCL Cancer Trial Centre (UCL CTC), acting on behalf of the sponsor UCL, will need to ensure all documents and requirements are reviewed and approved before activating the trial and sites.

\section{Intervention}

In the intervention arm, the patients will receive up to another two cycles of the same chemotherapy regime as the control arm, followed by RRT within 2-6 weeks of day 1 of cycle 4 of chemotherapy. RRT can be delivered to the primary lung tumour either by conventional radiotherapy or SABR, if appropriate, followed by SABR/SRS to the oligometastatic lesion(s) (figure 3). Maintenance chemotherapy is permitted according to local practice.

The radiotherapy must be performed by an approved site principal investigator who is experienced in treating NSCLC. Patients will be planned and treated per the SARON RT Planning and Delivery Guidelines (online supplementary appendix 1).

\section{Safety monitoring}

The treating physician will be able to modify or discontinue a patient treatment in either arm for various reasons, including perceived harm or toxicities. The treating physician will have to enter protocol deviations, treatment interruptions and adverse toxicities in the case report form (CRF) and submit it to the UCL CTC. 


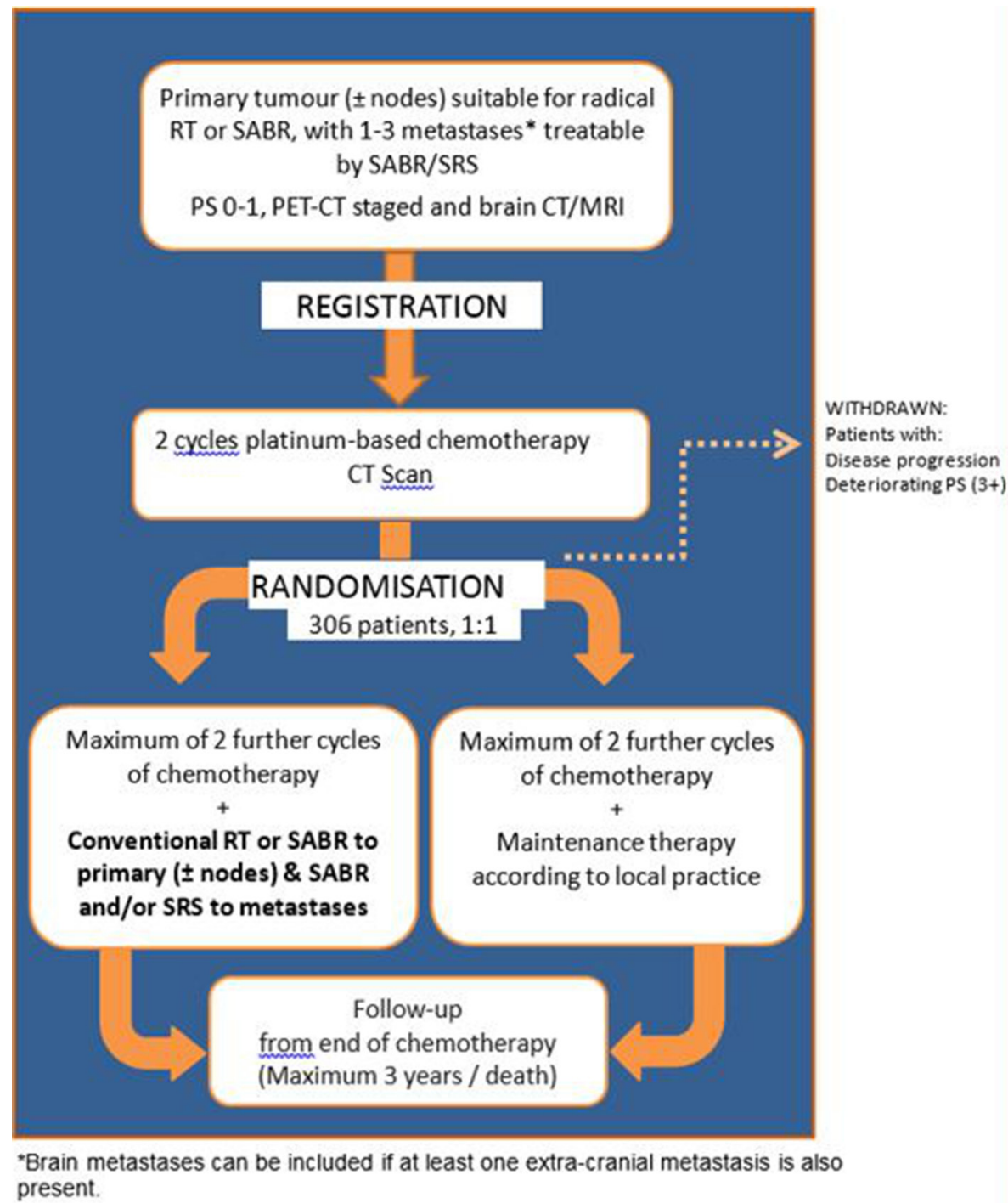

Figure 2 SARON trial schema. RT, radiotherapy; SABR, stereotactic ablative radiotherapy; SRS, stereotactic radiosurgery.

All serious adverse events must be reported to UCL CTC within 24 hours. The Trial Management Group (TMG) and Independent Data Monitoring Committee (IDMC) will also adopt a safety monitoring role and will review safety issues. Protocols amendments if required will be disseminated to all relevant parties.
Initial feasibility substudy

The aims of the feasibility substudy are:

- To satisfy the TMG and IDMC that recruitment targets are likely to be met for the remainder of the main trial.

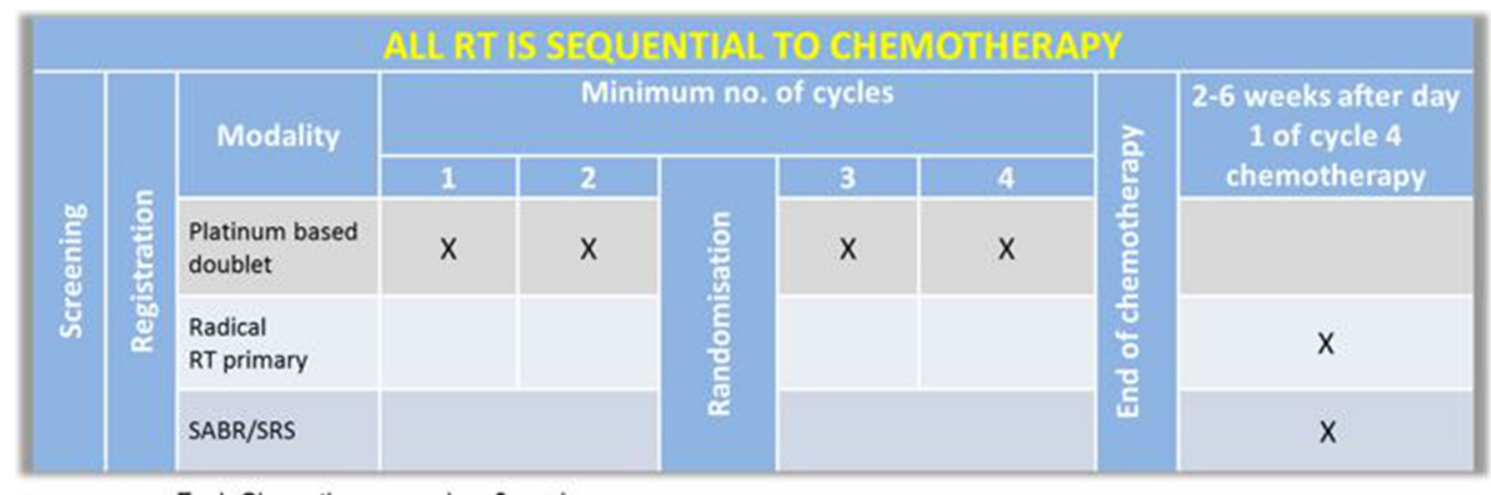

Each Chemotherapy cycle $=3$ weeks

Figure 3 Summary of treatment in investigational arm. RT, radiotherapy; SABR, stereotactic ablative radiotherapy; SRS, stereotactic radiosurgery. 


\begin{tabular}{|ccc|}
\hline $\begin{array}{c}\text { Number of } \\
\text { patients }^{*}\end{array}$ & $\begin{array}{c}\text { Number of G3+ } \\
\text { RTPN }\end{array}$ & Action \\
\hline \multirow{2}{*}{20} & 11 or more & Stop \\
\cline { 2 - 3 } & $2-10$ & Continue for another 10 patients \\
Safe to continue
\end{tabular}

${ }^{\star}$ On the Investigational Arm only. The initial estimate is that only 20 patients will be required. If more are required then the pilot study will be extended according to the numbers provided in the table

** Up until 3 months post SABR

Figure 4 Table detailing number of patients required with $\geq$ grade 3 to action a stoppage on treatment of thoracic metastases SABR. RTPN, radiation-induced pneumonitis; SABR, stereotactic ablative radiotherapy.

- To determine that the dropout/progression rate in the first 50 randomised patients is $<30 \%$ (ie, for at least 50 patients randomised and treated, no more than 72 patients have been registered).

- That there are no major logistical problems identified in the delivery of SABR/SRS.

Trial recruitment will not be suspended during the period of feasibility assessment.

As well as the aims above, the feasibility assessment will assess:

- Practicality of achieving recruitment targets

- Compare actual recruitment rate with expected recruitment rate.

- Explore reasons for screening failures/ineligibility.

- Review drop-off rate between registration and randomisation, including PFS rate following two cycles of chemotherapy prior to randomisation.

Logistics of delivering the investigational treatment

- Practicalities associated with delivering SABR/SRS, including:

- Technicalities in the QA.

- Access to SABR/SRS for all recruiting centres.

- Funding.

- Potential for contamination, as patients may seek $\mathrm{SABR} / \mathrm{SRS}$ if randomised to the chemotherapy only arm.

\section{Thoracic SABR safety substudy}

Patients treated with SABR to their thoracic metastases (including lung and other intrathoracic metastases, that is, thoracic spine and rib) will be monitored more closely for toxicity (figure 4 ). The first 20 patients in the experimental arm with thoracic metastases (these patients can also have non-thoracic metastases) will be treated and followed up for 3 months to assess adverse events. Until confirmation of safety, all thoracic metastases will be treated in one of approximately 10 centres, selected by the following criteria:

- Most treatment platforms (ie, manufacturers) are represented.

- All geographical locations of the UK have representation.

- An existing active SABR/SRS clinical programme is in place.

\section{Eligibility criteria}

Inclusion criteria

1. Patient $\geq 18$ years.

2. Histologically or cytologically confirmed NSCLC.

3. Staging with FDG PET-CT whole body scan and MRI brain or CT brain scan with IV contrast within 42 days prior to registration.

4. ECOG performance status $0-1$ at time of registration.

5. Patient presenting with synchronous primary disease and oligometastatic disease.

6. Patient is fit to receive four cycles of platinum-based doublet chemotherapy, cisplatin or carboplatin, as per local guidelines and assessment.

7. Primary tumour and involved nodes included in the radical RT volume must be suitable for radical RT (either conventional RT or SABR) — conventional RT fields do not need to be contiguous.

8. Patient is deemed fit to receive conventional RT and $\mathrm{SABR} / \mathrm{SRS}$ as per local guidelines and assessment.

9. Between one and three metastatic lesions, assessable as per RECIST V.1.1 and suitable for SABR and/or SRS (only one site of metastases OR the primary tumour needs to be measurable as per RECIST V.1.1).

i. Nodes included in the radical RT volume will not count towards the number of sites of metastases. 
Table 2 Acceptable brain metastases diameters

Second metastasis

Largest lesion diameter

diameter

$3 \mathrm{~cm}$ $\leq 2 \mathrm{~cm}$

$2.8 \mathrm{~cm}$ $\leq 2.4 \mathrm{~cm}$

$2.6 \mathrm{~cm}$ $\leq 2.6 \mathrm{~cm}$

ii. Nodes not treated in the radical RT volume are counted as metastases. The patient, though, must have stage IV disease. The same RT dose constraint eligibility criteria will apply to these nodes as to other metastases.

iii. Lower cervical supraclavicular lymph nodes and sternal notch nodes are considered N3. Higher neck nodes are considered as metastases (IASLC 2009 criteria).

10. Note: If brain metastasis present, at the time of randomisation, the following parameters must be met (table 2).

11. Acceptable lung function for radical lung radiotherapy as assessed according to local policy. Note: Potential thoracic substudy patients will need to complete pulmonary function tests preregistration.

12. No relevant comorbidities, including pulmonary fibrosis and connective tissue disorders.

\section{Further inclusion notes}

- For bone metastases, pre-SABR stabilisation should be considered as clinically appropriate. This does not exclude the patient from the study.

- For patients with brain metastases, there must be another metastatic lesion at another site to be eligible for the study (sum of intracranial and extracranial metastases must be $\leq 3$ ), as patients with brain only metastases would naturally be offered SRS/surgery to their intracranial disease in accordance with the published commissioning criteria for SRS set by NHS England.

- Patients with lung cancer and an additional malignant nodule are difficult to categorise, and the current stage classification rules are unclear. Such patients should be evaluated by the local multidisciplinary team to determine whether the additional lesion represents a second primary lung cancer or an additional tumour nodule corresponding to the dominant cancer. The SARON TMG will accept local MDM decisions on this and will aim to centrally review all baseline PET-CT, chest/abdomen CT scans (if performed) and brain MRI scans.

\section{Exclusion criteria}

1. Patient has had palliative radiotherapy to any tumour site prior to registration or requires palliative radiotherapy prior to randomisation.
2. Presence of EGFR or ALK/ROS-1 mutation (EGFR and ALK/ROS-1 testing is only mandatory for patients with adenocarcinoma).

3. One or more metastases previously treated with alternative ablative treatment, for example, RFA or surgery.

4. Patient has received any previous treatment for this NSCLC malignancy.

5. Patients who present with brain metastasis only and no sites of extracranial metastatic disease, that is, the presence of more than two brain metastases is an exclusion criterion.

6. Metastasis in sites where normal radiotherapy constraints cannot be met.

7. Brain metastasis within the brainstem.

8. Patients who have more than three metastases prior to trial registration.

9. Primary tumour or metastases causing direct invasion or high clinical suspicion of direct invasion of the wall of a major blood vessel.

10. Malignant pleural or pericardial effusion.

11. Patients with bilateral adrenal metastases.

12. History of prior malignant tumour likely to interfere with the protocol treatment or comparisons, unless the patient has been without evidence of disease for at least 3 years or the tumour was a non-melanoma skin tumour or early cervical cancer.

13. Women who are pregnant or breast feeding.

14. Stage III disease even with extensive nodal disease (ie, N3 nodal disease but no distant metastases).

15. Leptomeningeal disease.

\section{Eligibility criteria for randomisation}

Following cycle 2 of chemotherapy, patients must meet the following eligibility criteria for randomisation:

- No disease progression on postcycle 2 CT (as per RECIST V.1.1)

- Patients who cannot be randomised, only progression and OS data will be collected for these patients.

- Patients with no or less visible metastases following two cycles of chemotherapy are still eligible for randomisation

- If randomised to the investigational arm, these patients will receive $\mathrm{SABR} / \mathrm{SRS}$ to visible and $\mathrm{SABR} /$ SRS on relapse of existing metastases.

- Patients who experience progression with new metastases are not eligible for randomisation or for trial treatment.

- Patients with complete response of the lung primary following two cycles of chemotherapy are eligible for randomisation. If randomised to the Investigational Arm, these patients will still receive conventional RT to the site of the original primary tumour per protocol.

- ECOG Performance Status 0-2.

- Continued suitability for trial treatment as deemed by the treating clinician. 
TRIAL ENDPOINTS

\section{Main trial}

Primary endpoint

- OS: Measured from date of randomisation until date of death from any cause.

\section{Secondary endpoints}

- PFS: Time from randomisation until progression or death from any cause. Progression will be assessed using RECIST V.1.1.

- LC of primary and all metastases: This refers to tumours present at randomisation. Assessed as progression at one or more of these tumour sites.

- New distant metastasis-free survival: Time from randomisation until presence of new distant metastasis (ie, the emergence of tumour on imaging at anatomical sites where cancer was not present at the time of diagnosis) or death from any cause.

- Quality of life: As measured by EORTC QLQ-C30 ${ }^{34}$ and LC13. ${ }^{35}$

- Adverse events, assessed using CTCAE V.4.03, and dose delays, reduction and compliance to trial treatment.

\section{Feasibility substudy}

Primary endpoint

- Recruitment rate, logistical challenges, contamination, as patients may seek SABR/SRS outside the trial if randomised to the chemotherapy only Arm.

\section{Thoracic SABR safety substudy}

Primary endpoint

- Grade 3-5 radiation-induced pneumonitis (per CTCAE V.4.03) up until 3 months' post-thoracic SABR.

\section{Secondary endpoint}

- Other grade 3-5 RT-related toxicities (per CTCAE V.4.03).

\section{PARTICIPANT RECRUITMENT AND TIMELINE Sample size and study duration}

In total, 340 patients are required (170 per arm) to detect an improvement in median survival from 9.9 to 14.3 months, that is, a HR of at least 0.69 , with a two-sided $5 \%$ alpha and $85 \%$ power and a $10 \%$ dropout rate.

Prior to study initiation, a survey was sent to all UK radiotherapy centres on their SABR/SRS practices. At least 26 centres replied with an average of 4.6 suitable study patients in the previous 6 months. Assuming only half of this amount would satisfy the eligibility criteria, this would equate to around 120 patients. We thus expect that the trial will take around 3 years to accrue. We hope to include more than 30 centres in our study in improve on trial participation. Nevertheless, an early feasibility study will be conducted after the first 50 patients. The study opened August 2016.

\section{Recruitment and consent}

Patients will be identified through MDT meetings and clinic appointments. At the first consult, if all eligibility criteria are fulfilled, the team will introduce the trial and provide the patient information sheet (detailing rationale, trial design and risks involved) along with the relevant contact information. Participants will have at least 24 hours to consider participation. Consent will have to be obtained before collection of baseline tumour and demographic data and the QoL survey. Patients can withdraw at any stage of the trial.

After the second chemotherapy cycle, a CT scan will be performed. If prerandomisation eligibility criteria are fulfilled, the patient will be randomised. At this stage, patients who are unable to fulfil the criteria will be withdrawn from the study and only progression and OS data will be collected.

\section{Timeline}

During the treatment, all recruitment patients will be followed up with a blood test and physical examination before each chemotherapy treatment cycle. Patients in the interventional arm will have fortnightly reviews if they need conventional RT and weekly reviews when they have SABR/SRS.

On completion of fourth cycle of chemotherapy, an assessment will be done by the treating physician every month for the first 3 months, then 3 monthly until 2 years, then 6 monthly until 3 years postchemotherapy. During each scheduled visit, the patient will be assessed for disease status, performance status, adverse events and QoL questionnaire. A CT scan will be required at 3, 6, 12, 18, 24 and 36 months postchemotherapy (follow-up schedule in online supplementary appendix 2).

Patients in the Thoracic SABR Safety substudy will need lung function test at 12 and 24 months follow-up visits. Additionally, they will have fortnightly reviews for the 1st month after radiotherapy and an additional visit 3 months after radiotherapy.

Patient participation will be completed at death, study withdrawal or after 3 years of follow-up.

\section{DATA MANAGEMENT AND ANALYSIS \\ Data management}

Each participant is assigned a trial number by UCL CTC. The patient data including CRFs, supporting documents (eg, CT scan images, pathology reports) will be submitted to UCL CTC without any patient-specific identifiers to maintain confidentiality. To avoid missing data, forms are checked onsite before submission to UCL CTC. UCL CTC will check data for legibility, completeness, accuracy and consistency. Query reports will be sent to the data contact at site for checks and rectification.

Patients, who discontinue treatment for any reason, should be kept on trial for purpose of follow-up and data analysis. In patients lost to follow-up, every effort should 
be made by the site to contact the GP for information on patient status.

All trial-related documents will be archived centrally in a secure place for a minimum of 5 years at the end of the trial.

\section{Analysis}

The data will be analysed on completion of the study. Analysis of primary and secondary endpoints will be performed on an intention-to-treat population. The OS will also be conducted on per-protocol basis. Survival endpoints will be assessed using Cox proportional hazards models to estimate HRs and Cox regression analysis to adjust for effect on stratification factors. Missing data will be censored at the date they were last known to be alive. For all tests, we will use two-sided $p$ values with 5\% level of significance. QoL data will be analysed using mixed effects repeated measured measures model and reported by each domain. Adverse events (grade 3-5 CTCAE V.4.03 events) will be compared between groups using $\chi^{2}$ of Fisher's exact test for all patients who received any trial treatment.

\section{Thoracic SABR safety substudy analysis}

After the initial 20 patients with thoracic metastases have been treated and monitored for adverse events. If the data show evidence that the true rate of grade $3-5$ pneumonitis related to RT is above $30 \%$, the IDMC may consider suspending the treatment of thoracic metastases with SABR. If incidence is below $30 \%$, the treatment is safe to continue. If inconclusive (ie, $95 \%$ the CI of events crosses $30 \%$ ) the substudy will continue for another 10 patients until a conclusion can be drawn (figure 4).

\section{ETHICS AND DISSEMINATION}

Version 1.0 of the protocol was approved by West Midlands, Coventry \& Warwickshire Research Ethics Committee (Registration 15/WM/0392) on 7 December 2015. All participants will be given a SARON trial patient information sheet and will be required to give written informed consent. The SARON trial is supported by Cancer Research UK (C13530/A18015) and sponsored by UCL and coordinated by UCL CTC. Results will be submitted for presentation at local and international medical conference and expected to be published in a peer-reviewed journal.

\section{DISCUSSION}

There is growing evidence supporting the safety of SABR/SRS, its effect on LC and a possible impact on OS to warrant a randomised phase III trial. The key question regarding the successful completion of such a trial relates to its feasibility, which will be governed by patient numbers and access to appropriate SABR/SRS treatment. There are also no definitive data yet on the toxicity of $\mathrm{SABR} / \mathrm{SRS}$ in the specific context of thoracic SABR with radical RT to the primary tumour and mediastinal lymph nodes. SARON is an important study, as it will test the impact of radical RT and SABR/SRS on OS in oligometastatic NSCLC with an early evaluation of overall feasibility and toxicity for thoracic metastases. The study is currently recruiting; the study opened to recruitment in the UK on 11 August 2016 and the first patient was enrolled 19 August 2016.

\section{Author affiliations}

${ }^{1}$ St Bartholomew's Hospital, London, UK

${ }^{2}$ Cancer Research UK \& UCL Cancer Trials Centre, London, UK

${ }^{3}$ University Hospital Southampton NHS Foundation Trust, Southampton, UK

${ }^{4}$ National Radiotherapy Trials QA Group (RTTQA), Mount Vernon Hospital, Northwood, UK

${ }^{5}$ The University of Manchester and The Christie NHS Foundation Trust, Manchester, UK

${ }^{6}$ Department of Oncology, University of Oxford, Oxford, UK

${ }^{7}$ University College London Hospital, London, UK

${ }^{8}$ Centre for Cancer Research and Cell Biology, Queen's University of Belfast, Belfast, UK

${ }^{9}$ Addenbrooke's Hospital, Cambridge, UK

${ }^{10}$ The Clatterbridge Cancer Centre, Liverpool, UK

${ }^{11}$ Guys \& St Thomas NHS Trust, London, UK

${ }^{12}$ Department of Oncology, University College London, London, UK

Contributors DL conceived of the study and is the grant holder. DL, JC, ATB, CF-F, JF, MF, GGH, PM, SM, SH, NC, YN, DE, RP and BC initiated, developed and planned the study design. $\mathrm{NC}$ also provided statistical expertise in clinical trial design. RP and $\mathrm{DE}$ also developed the radiotherapy quality assurance programme for the trial. All the authors contributed to refinement of the study protocol and approved the final manuscript. All contributors have been heavily involved in the design of the trial and have been involved in the drafting and review of the article to be published.

Funding This research is funded by Cancer Research UK's (CR UK) Clinical Trials Awards and Advisory Committee (CTAAC). Grant reference number C13530/A18015.

Competing interests None declared.

Patient consent Detail has been removed from this case description/these case descriptions to ensure anonymity. The editors and reviewers have seen the detailed information available and are satisfied that the information backs up the case the authors are making.

Ethics approval West Midlands, Coventry \& Warwickshire Research Ethics Committee (15/WM/0392).

Provenance and peer review Not commissioned; externally peer reviewed. Data sharing statement Results/data not yet generated.

Open access This is an open access article distributed in accordance with the Creative Commons Attribution 4.0 Unported (CC BY 4.0) license, which permits others to copy, redistribute, remix, transform and build upon this work for any purpose, provided the original work is properly cited, a link to the licence is given, and indication of whether changes were made. See: https://creativecommons.org/ licenses/by/4.0/.

\section{REFERENCES}

1. Scagliotti GV, Parikh P, von Pawel J, et al. Phase III study comparing cisplatin plus gemcitabine with cisplatin plus pemetrexed in chemotherapy-naive patients with advanced-stage non-small-cell lung cancer. J Clin Oncol 2008;26:3543-51.

2. Lee SM, Rudd R, Woll PJ, et al. Randomized double-blind placebocontrolled trial of thalidomide in combination with gemcitabine and Carboplatin in advanced non-small-cell lung cancer. J Clin Oncol 2009;27:5248-54.

3. Oh Y, Taylor S, Bekele BN, et al. Number of metastatic sites is a strong predictor of survival in patients with nonsmall cell lung cancer with or without brain metastases. Cancer 2009;115:2930-8.

4. Pirker R, Pereira JR, Szczesna A, et al. Prognostic factors in patients with advanced non-small cell lung cancer: data from the phase III FLEX study. Lung Cancer 2012;77:376-82. 
5. Albain KS, Crowley JJ, LeBlanc M, et al. Survival determinants in extensive-stage non-small-cell lung cancer: the Southwest Oncology Group experience. J Clin Oncol 1991;9:1618-26.

6. Hellman S, Weichselbaum RR. Oligometastases. J Clin Oncol 1995;13:8-10.

7. Hanna GG, Landau D. Stereotactic body radiotherapy for oligometastatic disease. Clin Oncol 2015;27:290-7.

8. Siva S, MacManus M, Ball D. Stereotactic radiotherapy for pulmonary oligometastases: a systematic review. J Thorac Oncol 2010:5:1091-9.

9. Gerszten PC, Mendel E, Yamada Y. Radiotherapy and radiosurgery for metastatic spine disease: what are the options, indications, and outcomes? Spine 2009;34(22 Suppl):S78-92.

10. Lee MT, Kim JJ, Dinniwell R, et al. Phase I study of individualized stereotactic body radiotherapy of liver metastases. J Clin Oncol 2009;27:1585-91.

11. Parikh RB, Cronin AM, Kozono DE, et al. Definitive primary therapy in patients presenting with oligometastatic non-small cell lung cancer. Int J Radiat Oncol Biol Phys 2014;89:880-7.

12. Méndez Romero A, Wunderink W, Hussain SM, et al. Stereotactic body radiation therapy for primary and metastatic liver tumors: a single institution phase i-ii study. Acta Oncol 2006;45:831-7.

13. Kim MS, Cho CK, Yang KM, et al. Stereotactic body radiotherapy for isolated paraaortic lymph node recurrence from colorectal cancer. World J Gastroenterol 2009;15:6091-5.

14. Bignardi M, Cozzi L, Fogliata A, et al. Critical appraisal of volumetric modulated arc therapy in stereotactic body radiation therapy for metastases to abdominal lymph nodes. Int J Radiat Oncol Biol Phys 2009;75:1570-7.

15. Jereczek-Fossa BA, Fariselli L, Beltramo G, et al. Linac-based or robotic image-guided stereotactic radiotherapy for isolated lymph node recurrent prostate cancer. Radiother Oncol 2009;93:14-17.

16. Holy R, Piroth M, Pinkawa M, et al. Stereotactic body radiation therapy (SBRT) for treatment of adrenal gland metastases from nonsmall cell lung cancer. Strahlenther Onkol 2011;187:245-51.

17. Torok J, Wegner RE, Burton SA, et al. Stereotactic body radiation therapy for adrenal metastases: a retrospective review of a noninvasive therapeutic strategy. Future Oncol 2011;7:145-51.

18. Kang JK, Kim MS, Kim JH, et al. Oligometastases confined one organ from colorectal cancer treated by SBRT. Clin Exp Metastasis 2010;27:273-8.

19. Milano MT, Katz AW, Okunieff P. Patterns of recurrence after curativeintent radiation for oligometastases confined to one organ. Am J Clin Oncol 2010;33:157-63.

20. Salama JK, Hasselle MD, Chmura SJ, et al. Stereotactic body radiotherapy for multisite extracranial oligometastases: final report of a dose escalation trial in patients with 1 to 5 sites of metastatic disease. Cancer 2012;118:2962-70.

21. De Ruysscher D, Wanders R, van Baardwijk A, et al. Radical treatment of non-small-cell lung cancer patients with synchronous oligometastases: long-term results of a prospective phase II trial (Nct01282450). J Thorac Oncol 2012;7:1547-55.

22. Song CW, Kim MS, Cho LC, et al. Radiobiological basis of SBRT and SRS. Int J Clin Oncol 2014:19:570-8.

23. Gomez DR, Blumenschein GR, Lee JJ, et al. Local consolidative therapy versus maintenance therapy or observation for patients with oligometastatic non-small-cell lung cancer without progression after first-line systemic therapy: a multicentre, randomised, controlled, phase 2 study. Lancet Oncol 2016;17:1672-82.

24. lyengar $\mathrm{P}$, Wardak Z, Gerber DE, et al. Consolidative Radiotherapy for Limited Metastatic Non-Small-Cell Lung Cancer: A Phase 2 Randomized Clinical Trial. JAMA Oncol 2018;4:e173501.

25. Rusthoven KE, Kavanagh BD, Burri SH, et al. Multi-institutional phase I/II trial of stereotactic body radiation therapy for lung metastases. J Clin Oncol 2009;27:1579-84.

26. Ernst-Stecken A, Lambrecht U, Mueller R, et al. Hypofractionated stereotactic radiotherapy for primary and secondary intrapulmonary tumors: first results of a phase 1/II study. Strahlenther Onkol 2006;182:696-702.

27. Herfarth KK, Debus J, Lohr F, et al. Stereotactic single-dose radiation therapy of liver tumors: results of a phase I/II trial. J Clin Oncol 2001;19:164-70.

28. Rusthoven KE, Kavanagh BD, Cardenes $\mathrm{H}$, et al. Multi-institutional phase I/II trial of stereotactic body radiation therapy for liver metastases. J Clin Oncol 2009;27:1572-8.

29. Chawla S, Chen Y, Katz AW, et al. Stereotactic body radiotherapy for treatment of adrenal metastases. Int $J$ Radiat Oncol Biol Phys 2009;75:71-5.

30. Hoyer M, Roed H, Traberg Hansen A, et al. Phase II study on stereotactic body radiotherapy of colorectal metastases. Acta Oncol 2006;45:823-30.

31. Milano MT, Katz AW, Muhs AG, et al. A prospective pilot study of curative-intent stereotactic body radiation therapy in patients with 5 or fewer oligometastatic lesions. Cancer 2008;112:650-8.

32. Stinauer MA, Kavanagh BD, Schefter TE, et al. Stereotactic body radiation therapy for melanoma and renal cell carcinoma: impact of single fraction equivalent dose on local control. Radiat Oncol 2011;6:34.

33. Shaw AT, Kim DW, Nakagawa K, et al. Crizotinib versus chemotherapy in advanced ALK-positive lung cancer. N Engl J Med 2013;368:2385-94.

34. Aaronson NK, Ahmedzai S, Bergman B, et al. The European Organization for Research and Treatment of Cancer QLQ-C30: a quality-of-life instrument for use in international clinical trials in oncology. J Natl Cancer Inst 1993;85:365-76.

35. Bergman B, Aaronson NK, Ahmedzai S, et al. The EORTC QLQLC13: a modular supplement to the EORTC Core Quality of Life Questionnaire (QLQ-C30) for use in lung cancer clinical trials. EORTC Study Group on Quality of Life. Eur J Cancer 1994;30A:635-42. 